Short Communication

\title{
HOPPING IN BIRDS: IS THE CHOICE OF GAIT INFLUENCED BY CERVICAL MOBILITY AND FIELD OF VISION ?
}

\author{
Arunachalam Kumar \\ Professor \& Head, Department of Anatomy, K.S. Hegde M edical Academy, Nitte University, \\ Mangalore - 575 018, India. \\ Correspondence: \\ Arunachalam Kumar \\ E-mail: editornujhs@ nitte.edu.in
}

\begin{abstract}
:
One of the more intriguing questions in avian locomotion is why some birds, when on ground, choose to hop while others prefer walking. Biped gait is common to birds as well as the most evolved among mammals, man. Observations made show that, choice of gait in birds is determined by a remote factor - the range and extent of neck mobility. The wider the gamut of cervical mobility, the wider is the 'field of vision' available. Cervical movement capability is perhaps the single most deterministic factor in the bird's choice of terrestrial gait.
\end{abstract}

Keywords: bipedal gait, avifauna, visual range, cervical vertebrae, hopping.

\section{Introduction:}

One of the earliest bipeds to survive to modern era, are birds. Birds are unique in that many species among them have capability of flying in air, walking on land and swimming in water. The talent to exploit all three mediums, air, land and water for mobility makes birds occupy special in the evolutionary tree.

The biped gait in birds has thrown up variants. While do some birds walk while some others hop? This rather simple question throws up quite interesting and debatable answers. This brief article proposes that extra mobility of neck produced by wider cervical intervertebral articular range, in turn causes the bird to augment its visual range, which in turn allows the bird to progress slowly step by step. In birds with limited neck movement (reduced cervical intervertbral articular capacity) the biped gait is modified into hopping instead of striding. Hopping allows such birds to pivot round or Access this article online

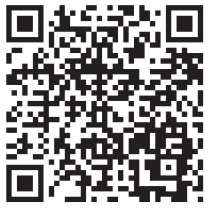
turn abruptly. In essence, the choice of gait, hopping or walking, is determined by visual field - which in turn is controlled by mobility range of neck'.

\section{Observations \& Discussion :}

Observations on biped mobility on birds and their locomotion reveal that, generally smaller birds hop while larger ones stride, strut or walk. Many theories and hypotheses float around the word of ornithology and kinematics on the how and why of avian locomotion.

a) Raptors (kites, eagles, hawks, owls) or such other birds, hop and strut far less than their arboreal and more terrestrial cousins, the passerines.

b) Arboreal and terrestrial, or low flying birds ground hop and walk more than the hi-fliers or nocturnal cousins

c) Birds that are endowed with a better degree of cervical (neck) vertebral rotation hop less. That is, birds that have limited swivel of neck (up to $180^{\circ}$ or less) hop more and more frequently than the birds that can rotate their heads through a much wider arc. Some birds have a complete or nearly complete 360 -degree field of view

Birds such as owls are gifted with nearly $270^{\circ}$ plus range of side to side swivel of neck vertebrae. The ability to rotate the head and increase field of vision to almost full circle range, makes the birds on alert to approaching danger - the talent to spot peril early, gives them the confidence to progress one step at a time (walk). 
Owls possess twice the number of cervical vertebrae, fourteen. The extra articulations in the cervix allows for more efficiency and fluidity (and range) in neck mobility. Birds also have more cervical vertebrae than many other animals; most have a highly flexible neck consisting of 1325 vertebrae ${ }^{2}$. Similar too is the gait long necked birds and raptors. In anatomical terms, the intervertebral cervical joints have wider articular surfaces of the plane / condlylar type in birds that soar, hover, or nocturnal. These birds, hypothetically at least, being gifted with a wider eye view and range of vision, thanks to an efficient cervical mobility, require less need to hop, twist and turn their whole bodies to visualize a wider field. They end up having to use their legs for walking. In less mobile necked birds, hopping, in lieu striding, allows shift of range and focus in field of vision

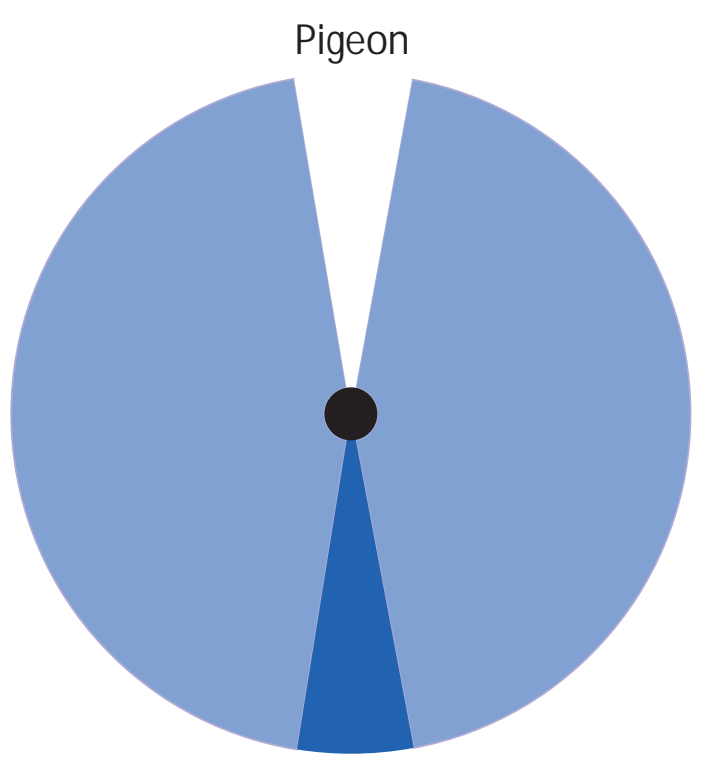

Binocular Vision

in continuously.

d) The natural processes of adaptation has perhaps given birds that strut and hop less with better cervical articular vertebral inputs, and vice versa, birds that can better hop, jump, strut and perch, need less efficiency in head rotation $\&$ mobility - the range and axis of movement of the cervical intervertebral joints accentuating or diminishing range of field of vision.

Prey species, like pigeons and robins, usually have a very wide field of view because they need to see danger coming from any direction. To achieve this most of their vision is monocular, like our peripheral vision, with only a narrow angle of binocular vision with good depth perception (Fig.l).

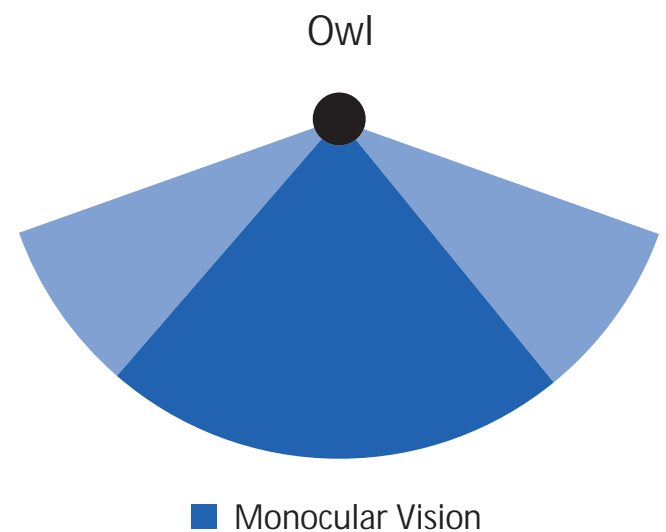

Fig. I: Showing range of vision in bids that 'walk' (pigeons and owls)

A pigeon can see nearly $360^{\circ}$ around its head, a real advantage when avoiding a peregrine. It's so important for birds to see what's coming, that some prey species can even move each eye independently!

Research into visual range and peripheral vision in some waders conclude that neck positioning plays a major role in success in foraging ${ }^{2}$.

Predator species usually have a narrower field of view because they need to have good depth perception in order to capture prey. The owl's field of view is more like ours with a wide area of binocular vision and narrow bands of peripheral, monocular vision on either side. Peregrines have fields of view similar to owls.

The chicken and the pigeon are two good examples of this. Such birds must judge distance by moving their heads and viewing an object with each eye independently, deducing from the displacement how far away the object must be ${ }^{3,4}$. 
The possibility of that neck mobility and visual ranges could be inter-dependent and complement type of gait used has not been thought of yet. Birds do not possess well developed musclesfor eyeball movement they rely on their ability to maneuver their head and neck for good visualization of objects ${ }^{4,5}$.

\section{References:}

1. Kumar JC\& Arunachalam Kumar; Biped locomotion: an evolutionary conundrum, Proceedings of the National Seminar on Bird Ecology \& Conservation, Eds. Varghese A, Sridhar S, Chakravarthy AK, Bhat HR \& Kumar P, Bangalore, 2005

2. Katzir G, Martin GR, (1994), Visual fields in herons (Ardeidae) panoramic vision beneath the bill; Naturwissenschaften, Vol. 81, Issue 4, pp 182-184

3. Kate St. John, http://www.wqed.org/birdblog/category/birdanatomy/

4. Sakas PS, Basic Avian Anatomy, http://www.4parrots.com/ Basic_Avian_Anatomy.htm

5. http://www.vetmed.vt.edu/education/curriculum/vm8054/eye/ binocs.htm
As these observations and conclusions derived are conjectural, speculative and hypothetical, the entire question of biomechanics and of neck as having bearing on bipedal kinetics may require a much deeper probe. Perimetrical visual analysis and collation of data on orbital fields in passerines, perchers, raptors and predators should aid in clarifying issues to some extent. 